\title{
Study on the Risk Factors of Postoperative Hypoxemia in Patients Undergoing Coronary Artery Bypass Grafting
}

\author{
Qiang Ji, MD, PhD; Yunqing Mei, MD, PhD; Xisheng Wang, MD, PhD; \\ Jing Feng, MD*; Jiangzhi Cai, MD, PhD; Yifeng Sun, MD; \\ Shiliang Xie, MD; Dawen Li, MD; Dayi Hu, MD, PhD*
}

\begin{abstract}
Background To investigate 576 patients undergoing coronary artery bypass grafting (CABG) and to evaluate independent high risk factors of postoperative hypoxemia following CABG.

Methods and Results The pre-, intra-, and post-operative materials in patients who had CABG performed on them from March 2004 to March 2008 in our hospital were analyzed retrospectively. The relative factors of postoperative hypoxemia were tested through descriptive analysis and logistic regression, and the independent risk factors were obtained. Among the 576 patients investigated, 156 cases suffered from postoperative hypoxemia, and the incidence rate of postoperative hypoxemia was $27.08 \%$. Through descriptive analysis and logistic regression, the independent risk factors of postoperative hypoxemia were as follows: preoperative chronic pulmonary diseases (odds ratio $(\mathrm{OR})=8.531,95 \%$ confidence interval $(\mathrm{CI}) 3.136-23.210$ ), preoperative acute myocardial infarction $(\mathrm{OR}=3.351,95 \%$ CI 1.539-7.296), and preoperative diabetes $(\mathrm{OR}=3.108,95 \% \mathrm{CI} 1.439-6.713)$. Preoperative acute myocardial infarction $(\mathrm{OR}=2.091,95 \%$ CI $1.520-4.416)$ is the independent risk factor during assisted ventilation after surgery, and preoperative chronic pulmonary diseases ( $\mathrm{OR}=7.19,95 \% \mathrm{CI} 2.807-18.413)$, pre-operative diabetes $(\mathrm{OR}=3.605,95 \% \mathrm{CI} 1.631-7.967)$, and preoperative acute myocardial infarction $(\mathrm{OR}=$ $3.604,95 \%$ CI $1.518-8.543$ ) are the 3 independent risk factors after decannulation following CABG.

Conclusions Preoperative chronic pulmonary diseases, preoperative acute myocardial infarction, and preoperative diabetes are 3 independent risk factors of postoperative hypoxemia following CABG. (Circ J 2008; 72: 1975-1980)
\end{abstract}

Key Words: Coronary artery bypass grafting; Etiology; Hypoxemia

C oronary artery bypass grafting (CABG) has been performed more extensively in China. With surgical improvement in technology, more and more elderly patients with coronary artery disease who have suffered with multiple organ dysfunction have had CABG been performed on them, and the postoperative hypoxemia seems to be on the increase. Some reports revealed that many factors and the interaction of these factors leads to postoperative hypoxemia following $\mathrm{CABG}^{1-3} \mathrm{In}$ this study, the pre-, intra-, and post- operative materials in patients with coronary artery disease who had CABG performed on them from March 2004 to March 2008 were collected and analyzed retrospectively. The relative risk factors were tested through descriptive analysis and logistic regression, the independent high risk factors of postoperative hypoxemia were obtained, and the aim is to prevent and treat postoperative hypoxemia after CABG.

(Received April 14, 2008; revised manuscript received June 18, 2008; accepted July 15, 2008; released online October 17, 2008)

Department of Thoracic Cardiovascular Surgery of Tongji Hospital of Tongji University, *Heart-Lung-Blood Vessel Center of Tongji University, Institute of Heart-Lung-Blood Vessel Disease of Tongji University, Shanghai, P. R. China

Mailing address: Yunqing Mei, MD, PhD, Department of Thoracic Cardiovascular Surgery of Tongji Hospital, Tongji University, 389 Xincun Rd, Shanghai200065, P.R.China. E-mail: rabbitmei2000@ yahoo.com.cn

All rights are reserved to the Japanese Circulation Society. For permissions, please e-mail: cj@j-circ.or.jp

\section{Methods}

\section{Patients}

From March 2004 to March 2007, 576 patients (412 male and 164 female, mean age 68.36 years) suffering from coronary artery disease had CABG performed on them in our hospital. Among these cases, 422 suffered from stable angina, and 154 instable angina. Coronary artery angiography revealed that double vessel disease was observed in 54 cases, triple vessel disease in 522 cases, and 150 cases were concurrent with left main trunk disease; 50 cases were concurrent with left ventricular aneurysm. The 382 cases were performed with a beating heart CABG (including 66 cases with cardiopulmonary bypass) and 194 cases with an onpump CABG. The number of bypass graftings ranged from 2 to 5 (mean 2.96 per case). The Swan-Ganz transcatheter was used to monitor hymodynamic parameters intra- and postoperatively. A blood gas analyzer (i-STAT Corporation, East Windsor, NJ, USA) was used to measure the arterial partial pressure of oxygen $\left(\mathrm{PaO}_{2}\right)$ and arterial oxygen saturation pre-, intra- and postoperatively. A Doppler-Ultrasound (VIVID 7) was used to measure the left ventricular ejection fraction (LVEF) and left ventricular endo-diastolic diameter (LVEDD) pre- and post-operatively.

\section{Methods}

The investigated materials were as follows: sex, age, smoking history and smoking index, preoperative acute cardiac infarction, preoperative hypertension, preoperative diabetes, preoperative chronic pulmonary diseases, pre- 


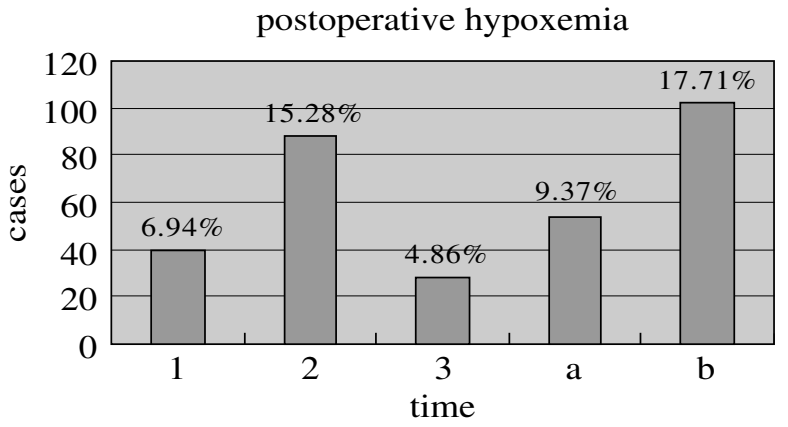

Fig 1. Time points of patients with postoperative hypoxemia in the study. Time point 1 , the first postoperative night; Time point 2 , the second postoperative night; Time point 3 , the third postoperative night; Time point a, before decannulation; Time point $\mathrm{b}$, after decannulation. $6.94 \%, 15.28 \%, 4.86 \%, 9.37 \%$, and $17.71 \%$ were the occurrence rates of postoperative hypoxemia for each of the corresponding time points.

operative LVEF and LVEDD, preoperative $\mathrm{PaO}_{2}$ (the value of preoperative $\mathrm{PaO}_{2}$ was obtained under no oxygen supply), preoperative left ventricular aneurysm, left main trunk disease, emergency operation, CABG with or without cardiopulmonary bypass, the duration of cardiopulmonary bypass and aortic cross-clamp, the number of diseased vessels and bypass graftings, the duration of the operation, intraoperative total liquid volume, and postoperative intubation time.
Criterion of Hypoxemia

On condition that mechanical ventilation $\left(\mathrm{FiO}_{2}\right.$ more than $45 \%$ ) or mask oxygen supply (more than $6 \mathrm{~L} / \mathrm{min}$ ) was administered following $\mathrm{CABG}, \mathrm{PaO}_{2}$ less than $[102-(0.33 \times$ age) $\mathrm{mmHg}$ is diagnosed as postoperative hypoxemia. Factors such as anemia, pain, anesthesia and so forth were excluded.

\section{Statistical Analysis}

Statistical analysis was performed using an SPSS10 statistical software package. All $\mathrm{p}$ values $<0.05$ were considered to indicate statistical significance. Each of the investigated materials were defined as independent variables; postoperative hypoxemia (or not) following CABG was defined as a dependent variable, and descriptive analysis was performed (the unpaired t-test or t'-test according to the homogeneity test for variance was used to compare measurement data, and chi-squared analysis was used to compare enumeration data). The risk factors that were obtained by descriptive analysis were tested through logistic regression, and the independent high risk factors were obtained.

\section{Results}

Patients

The 576 cases were included in the present study, and 156 cases developed postoperative hypoxemia, accounting for $27.08 \%$. Postoperative hypoxemia was observed mostly on the second postoperative night $(\mathrm{p}<0.05)$ and post-

Table 1 Incidence Rate of Postoperative Hypoxemia and Relative Factors

\begin{tabular}{|c|c|c|c|c|c|}
\hline Factor & $n$ & $\begin{array}{c}\text { Case } \\
\text { (hypoxemia) }\end{array}$ & $\begin{array}{c}\text { Incidence } \\
\text { rate }\end{array}$ & $\begin{array}{c}\text { Chi-square } \\
\text { value }\end{array}$ & $p$ value \\
\hline \multicolumn{6}{|l|}{ Gender } \\
\hline Male & 512 & 108 & $26.21 \%$ & \multirow{2}{*}{0.28} & \multirow{2}{*}{$>0.05$} \\
\hline Female & 164 & 48 & $29.27 \%$ & & \\
\hline \multicolumn{6}{|c|}{ Hypertension history } \\
\hline Yes & 312 & 102 & $32.69 \%$ & \multirow{2}{*}{5.42} & \multirow{2}{*}{$<0.05$} \\
\hline No & 264 & 54 & $20.45 \%$ & & \\
\hline \multicolumn{6}{|c|}{ Diabetes history } \\
\hline Yes & 142 & 58 & $40.85 \%$ & \multirow{2}{*}{9.04} & \multirow{2}{*}{$<0.01$} \\
\hline No & 434 & 98 & $22.58 \%$ & & \\
\hline \multicolumn{6}{|c|}{ COPD history } \\
\hline Yes & 64 & 36 & $56.25 \%$ & \multirow{2}{*}{15.51} & \multirow{2}{*}{$<0.001$} \\
\hline No & 512 & 120 & $23.44 \%$ & & \\
\hline \multicolumn{6}{|c|}{ Smoking history } \\
\hline Yes & 188 & 108 & $57.45 \%$ & \multirow{2}{*}{65.15} & \multirow{2}{*}{$<0.001$} \\
\hline No & 388 & 48 & $12.37 \%$ & & \\
\hline \multicolumn{6}{|c|}{ Acute myocardial infarction } \\
\hline Yes & 132 & 62 & $46.97 \%$ & \multirow{2}{*}{17.15} & \multirow{2}{*}{$<0.001$} \\
\hline No & 444 & 94 & $21.17 \%$ & & \\
\hline \multicolumn{6}{|c|}{ Left main trunk disease } \\
\hline Yes & 150 & 38 & $25.33 \%$ & \multirow{2}{*}{0.16} & \multirow{2}{*}{$>0.05$} \\
\hline No & 426 & 118 & $27.70 \%$ & & \\
\hline \multicolumn{6}{|c|}{ Left ventricular aneurysm } \\
\hline Yes & 50 & 22 & $44.00 \%$ & \multirow{2}{*}{3.97} & \multirow{2}{*}{$<0.05$} \\
\hline No & 526 & 134 & $25.48 \%$ & & \\
\hline \multicolumn{6}{|c|}{ Emergency operation } \\
\hline Yes & 48 & 12 & $25.00 \%$ & \multirow{2}{*}{0.06} & \multirow{2}{*}{$>0.05$} \\
\hline No & 528 & 144 & $27.27 \%$ & & \\
\hline \multicolumn{6}{|c|}{ Cardiopulmonary bypass } \\
\hline Yes & 260 & 90 & $34.62 \%$ & \multirow{2}{*}{6.81} & \\
\hline No & 316 & 66 & $20.89 \%$ & & $<0.01$ \\
\hline Aortic cros & & & & & \\
\hline Yes & 194 & 76 & $39.18 \%$ & 1083 & $<0001$ \\
\hline No & 382 & 80 & $20.94 \%$ & 10.83 & $<0.001$ \\
\hline
\end{tabular}

COPD, chronic obstructive pulmonary disease. 
Table 2 Relative Factors of Postoperative Hypoxemia Following CABG

\begin{tabular}{|c|c|c|c|c|c|}
\hline \multirow{2}{*}{ Parameters } & \multicolumn{2}{|c|}{ Hypoxemia group } & \multicolumn{2}{|c|}{ Non-hypoxemia group } & \multirow{2}{*}{$p$ value } \\
\hline & $n$ & Value of parameter & $n$ & Value of parameter & \\
\hline Age (years) & 156 & $68.62 \pm 6.94$ & 420 & $68.27 \pm 7.96$ & 0.3665 \\
\hline Smoking index & 48 & $975.00 \pm 751.38$ & 140 & $902.86 \pm 532.96$ & 0.3048 \\
\hline Vessel disease & 156 & $2.92 \pm 0.27$ & 420 & $2.90 \pm 0.30$ & 0.2760 \\
\hline Number of bypass graftings & 156 & $3.12 \pm 0.71$ & 420 & $2.90 \pm 0.69$ & 0.0102 \\
\hline Preoperative LVEF & 156 & $0.55 \pm 0.10$ & 420 & $0.58 \pm 0.09$ & 0.0114 \\
\hline Preoperative LVEDD & 156 & $52.95 \pm 9.55$ & 420 & $50.07 \pm 5.83$ & 0.0011 \\
\hline Preoperative $\mathrm{PaO}_{2}(\mathrm{mmHg})$ & 156 & $74.84 \pm 11.36$ & 420 & $77.43 \pm 10.09$ & 0.0313 \\
\hline Duration of $C P B(\mathrm{~min})$ & 90 & $116.60 \pm 22.96$ & 170 & $97.86 \pm 14.69$ & 0.0010 \\
\hline Duration of $A C C$ ( $\mathrm{min})$ & 76 & $68.81 \pm 48.78$ & 118 & $52.56 \pm 12.00$ & 0.0070 \\
\hline Duration of surgery (min) & 156 & $266.14 \pm 78.62$ & 420 & $260.00 \pm 67.64$ & 0.2567 \\
\hline Intraoperative liquid volume $(\mathrm{ml})$ & 156 & $4,083.33 \pm 1,562.92$ & 420 & $3,588.57 \pm 965.16$ & 0.0007 \\
\hline Intubation time $(h)$ & 156 & $20.86 \pm 16.14$ & 420 & $18.37 \pm 17.24$ & 0.1342 \\
\hline
\end{tabular}

$C A B G$, coronary artery bypass grafting; $L V E F$, left ventricular ejection fraction; $L V E D D$, left ventricular endo-diastolic diameter; $\mathrm{PaO}$, arterial partial pressure of oxygen; $C P B$, cardiopulmonary bypass; $A C C$, aortic cross-clamp.

Table 3 Comparison Between Pre and Postoperative $\mathrm{PaO}_{2}$

\begin{tabular}{lcccc}
\hline \hline & $n$ & $\begin{array}{c}\text { Preoperative } \mathrm{PaO}_{2} \\
(\mathrm{mmHg})\end{array}$ & $\begin{array}{c}\text { Postoperative } \mathrm{PaO}_{2} \\
(\mathrm{mmHg})\end{array}$ & $p$ value \\
\hline $\begin{array}{l}\text { No hypoxemia } \\
\text { Hypoxemia }\end{array}$ & 420 & $77.43 \pm 10.09$ & $85.69 \pm 19.82$ & 0.00002 \\
\hline
\end{tabular}

Abbreviation see in Table 2 .

Table 4 Logistic Regression of Relative Factors of Postoperative Hypoxemia

\begin{tabular}{lcccc}
\hline \hline & $\beta$ & $S_{\beta}$ & Chi-square value & OR $(95 \%$ CI $)$ \\
\hline Constant & -2.926 & 1.416 & 4.269 & 0.054 \\
Acute myocardial infarction & 1.209 & 0.397 & 9.284 & $3.351(1.539-7.296)$ \\
Diabetes history & 1.134 & 0.393 & 8.329 & $3.108(1.439-6.713)$ \\
COPD history & 2.144 & 0.511 & 17.622 & $8.531(3.136-23.210)$ \\
Preoperative $\mathrm{PaO}_{2}$ & 0.065 & 0.017 & 14.296 & $1.067(1.032-1.103)$ \\
Duration of $\mathrm{CPB}$ & -0.016 & 0.004 & 21.334 & $0.984(0.977-0.991)$ \\
Intraoperative liquid volume & -0.001 & 0.000 & 28.031 & $0.999(0.999-0.999)$ \\
\hline
\end{tabular}

OR, odds ratio; CI, confidence interval. Other abbreviations see in Tables 1,2.

decannulation $(\mathrm{p}<0.05)($ Fig 1$)$. Of 156 cases, 152 suffered from postoperative hypoxemia that ameliorated through respiratory assistance with trachea intubation or bi-level positive airway pressure ventilation; 1 case died of infective shock due to severe pulmonary infection, and 3 cases died of severe low cardiac output in despite of intra-aortic balloon pump and trachea intubation and assisted ventilation.

\section{Methods}

Descriptive analysis is presented in Tables 1,2. Table 1 showed that preoperative hypertension, preoperative diabetes, preoperative smoking, preoperative chronic pulmonary diseases, preoperative left ventricular aneurysm, cardiopulmonary bypass, and aortic cross-clamp might be risk factors of postoperative hypoxemia following CABG. Table 2 revealed that postoperative hypoxemia following CABG might be related significantly with preoperative LVEF and LVEDD, preoperative $\mathrm{PaO}_{2}$, number of bypass graftings, the duration of cardiopulmonary bypass and aortic cross-clamp, and intraoperative total liquid volume. In addition, the comparison between pre and postoperative $\mathrm{PaO}_{2}$ is presented in Table 3. Postoperative $\mathrm{PaO}_{2}$ was significantly lower than preoperative $\mathrm{PaO}_{2}$ in the hypoxemia group, whereas postoperative $\mathrm{PaO}_{2}$ was markedly higher than preoperative $\mathrm{PaO}_{2}$ in the non-hypoxemia group.
Statistical Analyses

Logistic regression (postoperative hypoxemia (or not) following $\mathrm{CABG}$ was defined as a dependent variable) is presented in Table 4. According to Table 4, the independent risk factors of postoperative hypoxemia following $\mathrm{CABG}$ might be as follows: preoperative chronic pulmonary disease $($ odds ratio $(\mathrm{OR})=8.531,95 \%$ confidence interval $(\mathrm{CI})$ 3.136-23.210), preoperative acute myocardial infarction (OR=3.351, 95\% CI 1.539-7.296), preoperative diabetes $(\mathrm{OR}=3.108,95 \%$ CI $1.439-6.713)$, preoperative $\mathrm{PaO}_{2}(\mathrm{OR}=$ $1.067,95 \%$ CI 1.032-1.103), intraoperative total liquid volume (OR=0.999, 95\% CI 0.999-0.999), and the duration of cardiopulmonary bypass $(\mathrm{OR}=0.984,95 \%$ CI 0.977 $0.991)$. And the regression equation used was: $Y=-2.926+$ $1.209 \times$ preoperative acute myocardial infarction $+1.134 \times$ preoperative diabetes $+2.144 \times$ preoperative chronic pulmonary disease $+0.065 \times$ preoperative $\mathrm{PaO}_{2}-0.016 \times$ duration of cardiopulmonary bypass $-0.001 \times$ intraoperative total liquid volume.

Logistic regression (postoperative hypoxemia (or not) during mechanical ventilation after surgery was defined as a dependent variable) is presented in Table 5. The independent risk factors of postoperative hypoxemia during mechanical ventilation after surgery might be as follows: preoperative acute myocardial infarction $(\mathrm{OR}=2.091,95 \%$ CI 1.520 
Table 5 Logistic Regression of Relative Factors of Postoperative Hypoxemia Before Decannulation

\begin{tabular}{lcccc}
\hline \hline & $\beta$ & $S_{\beta}$ & Chi-square value & OR (95\%CI) \\
\hline Acute myocardial infarction & 1.069 & 0.775 & 9.557 & $2.912(1.520-4.416)$ \\
Preoperative $\mathrm{PaO}_{2}$ & 0.187 & 0.045 & 16.908 & $1.205(1.103-1.317)$ \\
Duration of $\mathrm{CPB}$ & -0.049 & 0.01 & 23.81 & $0.953(0.934-0.971)$ \\
Intraoperative liquid volume & -0.002 & 0.001 & 16.098 & $0.998(0.997-0.999)$ \\
Constant & -2.394 & 2.262 & 0.223 & 0.091 \\
\hline
\end{tabular}

Abbreviations see in Tables 1,2,4.

Table 6 Logistic Regression of Relative Factors of Postoperative Hypoxemia After Decannulation

\begin{tabular}{lcccc}
\hline \hline & $\beta$ & $S_{\beta}$ & Chi-square value & OR (95\%CI) \\
\hline Acute myocardial infarction & 1.281 & 0.441 & 8.45 & $3.601(1.518-8.543)$ \\
Preoperative diabetes & 1.282 & 0.405 & 10.048 & $3.605(1.631-7.967)$ \\
Preoperative COPD & 1.973 & 0.48 & 16.903 & $7.19(2.807-18.413)$ \\
Preoperative PaO2 & 0.033 & 0.017 & 3.985 & $1.034(1.001-1.068)$ \\
Constant & -2.171 & 1.478 & 2.16 & 0.114 \\
\hline
\end{tabular}

Abbreviations see in Tables 1,2,4.

4.416), preoperative $\mathrm{PaO}_{2}(\mathrm{OR}=1.205$, 95\% CI $1.103-$ 1.317), intraoperative total liquid volume $(\mathrm{OR}=0.998$, 95\% CI 0.997-0.999), and the duration of cardiopulmonary bypass $(\mathrm{OR}=0.953,95 \% \mathrm{CI} 0.934-0.971)$.

In addition, logistic regression (postoperative hypoxemia (or not) after decannulation was defined as a dependent variable) is presented in Table 6 . The independent risk factors of postoperative hypoxemia after decannulation might be as follow: preoperative chronic pulmonary diseases $(\mathrm{OR}=$ $7.190,95 \%$ CI 2.807-18.413), preoperative diabetes ( $\mathrm{OR}=$ $3.605,95 \%$ CI 1.631-7.967), preoperative acute myocardial infarction (OR=3.601, 95\% CI 1.518-8.543), and preoperative $\mathrm{PaO}_{2}(\mathrm{OR}=1.034,95 \%$ CI 1.001-1.068).

\section{Discussion}

One of principles of monitoring patients with coronary artery disease following $\mathrm{CABG}$ is increasing oxygen supply and decreasing oxygen consumption. Postoperative hypoxemia following CABG prolongs the duration of endotracheal intubation and assisted ventilation, and increases the incidence rate of pulmonary infection, which might aggravate hypoxemia inversely. Most patients who underwent $\mathrm{CABG}$ were senile, and long-term myocardial ischemia damaged the cardiac function, and hypoxemia aggravated cardiac dysfunction. Myocardial oxygen consumption was increased by a fast heart rate caused by hypoxic tissue due to hypoxemia, and lactate accumulation can decrease myocardial function and make homodynamic unstable, induced arrhythmia. Postoperative hypoxemia prolongs the duration of ICU stay and increases patients' and society's economic burden. Thus, it is very important for clinicians to confirm the high risk factors of postoperative hypoxemia following $\mathrm{CABG}$, which is beneficial in terms of prevention and treatment of postoperative hypoxemia following CABG.

In this study, the incidence rate of postoperative hypoxemia was $27.08 \%$, which surpassed (slightly) the 18.80$23.64 \%$ found in other reports! $!^{-5}$ Through univariate analysis, the risk factors were as follows: preoperative hypertension, preoperative diabetes, preoperative chronic pulmonary diseases, preoperative smoking, preoperative acute myocardial infarction, preoperative ventricular aneurysm, preoperative LVEF, preoperative LVEDD, preoperative $\mathrm{PaO}_{2}$, number of bypass graftings, the duration of cardiopulmonary bypass and aortic cross-clamp, and intraoperative total liquid volume. Furthermore, through logistic regression, the independent risk factors of postoperative hypoxemia following CABG were as follows: preoperative chronic pulmonary diseases, preoperative acute myocardial infarction, and preoperative diabetes. Preoperative chronic pulmonary diseases were one of the key factors of postoperative hypoxemia following CABG. Small airway obstruction and larger physiologic dead space were observed in patients suffering from chronic pulmonary diseases; furthermore, shallow-quick breathing caused by various reasons after surgery was observed in the early postoperative period, which reduced pulmonary alveolar ventilation volume. Bronchospasm and bronchial stenosis induced by cough and sucking sputum might develop in patients suffering from chronic pulmonary diseases and preoperative hypoxemia, which contributed to obstructive ventilator disorder. Hyperventilation is also observed in patients with chronic pulmonary diseases; this could not be compensated to increase the tidal volume. The reduction, fragmentation and degeneration of elastic fiber might lead to attenuating pulmonary elastic recoil and decreasing the lung compliance in patients with chronic pulmonary diseases. The above-mentioned reasons can cause obstructive and restrictive ventilation disorder, and decrease vital capacity and maximal ventilation volume, 6 ,7 Chronic pulmonary diseases reduced pulmonary elastic recoil, the diameter of the air duct, and the number of lung capillary beds caused by damaging pulmonary alveoli, which led to ventilation/perfusion unbalance, and finally damaged the gas exchange function?

Preoperative acute myocardial infarction was also an important risk factor of postoperative hypoxemia following CABG. Myocardial edema was observed in patients with acute myocardial infarction, which led to low cardiac function. And patients with preoperative low cardiac function were prone to hypoxemia and even postoperative acute respiratory distress syndrome, which might be associated with low organ perfusion. Meanwhile, low cardiac function would increase pulmonary vascular resistance, pulmonary vascular permeability and pulmonary alveolar exudates, which made the diffusion disorder and ventilation-perfusion imbalance, and thus aggravating hypoxia,, 10

The decrease of lung function was considered to be associated with the course of diabetes, and tight glycemic con- 
trol might delay the deterioration of lung function. Some studies revealed that the lung was one of the "target organs" that diabetes attacked.1 The impairment of lung function might mainly be associated with microangiopathy caused by diabetes, which might be attributed to structural change of pneumoangiogram endothelial cell and the increase of pneumoangiogram permeability caused by hyperglycemia. In addition, the leakage of plasma and micromolecule protein increased and the glycosylation of tissue protein led to the accrementition of connective tissue, which decreased the elastance of the lung and affected the ventilation function further. The impairment of vessel epithelial cells led to a thickening of pulmonary capillary basal membrane and alveolar epithelium, which could cause diffusion dysfunction. Hyperglycemia might lead to the disorder of synthesis and secretion of alveolar surfactants, which could cause the collapse of the pulmonary alveoli, diminish the gas-exchange surface and aggravated diffusion dysfunction. Pulmonary microangiopathy was confirmed by pathohistology in diabetic patients, and included widespread alterations of basal lamina, hyperplasia of the extracellular matrix, and the change of ultramicrostructure. Thus, reasonable glycemic control preoperatively was crucial for surgery.

In addition, through logistic regression, the independent risk factors of postoperative hypoxemia following CABG might be also as follows: intraoperative total liquid volume and the duration of cardiopulmonary bypass. As the study indicated, there was close association between intraoperative total liquid volume and postoperative hypoxemia following $\mathrm{CABG}$. Increasingly, postoperative hypoxemia was observed with the duration of cardiopulmonary bypass and intraoperative total liquid volume. However, retention of liquid could not be measured merely by transfusion volume, because of urinary volume and amount of infusion included crystalloid and colloid. Cardiac diastolic function was lowered in most patients suffering from coronary artery disease, so the infusion volume should be appropriately limited during operation. The duration of cardiopulmonary bypass was also one of risk factors of postoperative hypoxemia following CABG. Cardiopulmonary bypass is associated with a whole body inflammatory response, which involves activation of complement, leukocytes, and endothelial cells with the secretion of cytokines, proteases, arachidonic acid metabolites, and oxygen free radicals; it will lead to acute lung injury. Previous studies have measured the plasma levels of elastase and complement $\mathrm{C} 3$ a for neutrophils destroyed and observed platelet degranulaton by comparing on-pump CABG with off-pump CABG, with the level in the off-pump CABG group lower than that in the on-pump CABG group!2,13 This therefore might shorten the duration of surgery, especially the duration of cardiopulmonary bypass, and might reduce the postoperative pulmonary complications. Advancing the surgeon skills and developing off-pump CABG were beneficial for patient recovery.

Many studies indicated that long-term smoking made patients prone to hypoxemia and ARDS after cardiac surgery3,14 However, smoking was not an independent risk factor of postoperative hypoxemia following CABG through this study. It might be relative with most patients with coronary artery disease and those who smoked. There was a high proportion of smokers in the non-hypoxemia group in this study, and there was almost a similar smoking index between the non-hypoxemia group and the hypoxemia group $(975.00 \pm 751.39$ per year in the hypoxemia group vs $902.86 \pm 532.96$ per year in the non-hypoxemia group) in this study. In contrast, smoking cessation periods had an influence on postoperative respiratory status (patients who had elective CABG surgery were required to stop smoking at least 2 weeks preoperatively in this study). In addition, many studies showed that senility was also an independent risk factor of postoperative hypoxemia following $\mathrm{CABG}$ ? There was no significant difference in the mean age between hypoxemia groups and non-hypoxemia group (68.62 \pm 6.94 years old vs $68.27 \pm 7.96$ years old). This might be relative with senile patients included in our study.

The study also investigated the incidence rate of postoperative hypoxemia before and after decannulation. The results indicated that the incidence rate of postoperative hypoxemia was significantly higher after decannulation compared with before decannulation $(17.71 \%$ vs $9.37 \%$, $\mathrm{p}<0.05)$. Through logistic regression, the independent risk factors of postoperative hypoxemia following CABG were not absolutely similar pre- and post- decannulation. Preoperative acute myocardial infarction was one important factor of postoperative hypoxemia before decannulation, and preoperative chronic pulmonary diseases, preoperative acute myocardial infarction and preoperative diabetes were 3 important risk factors of postoperative hypoxemia after decannulation. The reasons might be as follows: preoperative acute myocardial infarction caused low organ perfusion and low cardiac function, which caused a diffusion disorder and ventilation-perfusion imbalance through increasing pulmonary vascular resistance, pulmonary vascular permeability and pulmonary alveolar exudates. Despite preoperative acute myocardial infarction aggravating hypoxia, during mechanical ventilation following $\mathrm{CABG}$, a high flow of oxygen, large tidal volume, and positive end-expiratory pressure increased oxygenation and enshrouded the harm caused by preoperative acute myocardial infarction; after decannulation, the effect of chronic pulmonary diseases, acute myocardial infarction and diabetes on the lung gradually showed that there was a lack of assisted respiratory work, and the level of $\mathrm{PaO}_{2}$ presented a declining trend; with the medical and nurse interventions, the level of $\mathrm{PaO}_{2}$ might have gradually rebound. Thus, extubation indications of tracheal intubation should be emphasized, and respiratory tract nursing care should be strengthened.

\section{References}

1. Andreassen S, Rees SE, Kjaergaard S, Thorgaard P, Winter SM, Morgan CJ, et al. Hypoxemia after coronary bypass surgery modeled by resistance to oxygen diffusion. Crit Care Med 1999; 27: 24452453.

2. Frangos SG, Schwartz DR. Continuous positive airway pressure and postoperative hypoxemia. JAMA 2005; 293: 2714; author's reply $2714-2715$

3. Warner MA, Offord KP, Warner ME, Lennon RL, Conover MA, Jansson SU. Role of preoperative cessation of smoking and other factors in postoperative pulmonary complications: A blinded prospective study of coronary artery bypass patients. Mayo Clin Proc 1989; 64: $609-616$.

4. Squadrone V, Coha M, Cerutti E, Schellino MM, Biolino P, Occella $\mathrm{P}$, et al. Continuous positive airway pressure for treatment of postoperative hypoxemia: A randomized controlled trial. JAMA 2005; 293: $589-595$

5. Lundstrom LH, Nygard E, Hviid LB, Pedersen FM, Ravn J, Aldershvile J, et al. The effect of thoracic epidural analgesia on the occurrence of late postoperative hypoxemia in patients undergoing elective coronary bypass surgery: A randomized controlled trial. Chest 2005; 128: 1564-1570.

6. Filaire M, Bedu M, Naamee A, Aubreton S, Vallet L, Normand B, et al. Prediction of hypoxemia and mechanical ventilation after lung resection for cancer. Ann Thorac Surg 1999; 67: 1460-1465.

7. Christenson JT, Aeberhard JM, Badel P, Pepcak F, Maurice J, 
Simonet F, et al. Adult respiratory distress syndrome after cardiac surgery. Cardiovasc Surg 1996; 4: 15-21.

8. Xue FS, Li BW, Zhang GS, Liao X, Zhang YM, Liu JH, et al. The influence of surgical sites on early postoperative hypoxemia in adults undergoing elective surgery. Anesth Analg 1999; 88: 213-219.

9. Ferrari G, Olliveri F, De Filippi G, Milan A, Apra F, Boccuzzi A, et al. Noninvasive positive airway pressure and risk of myocardial infarction in acute cardiogenic pulmonary edema: Continuous positive airway pressure vs noninvasive positive pressure ventilation. Chest 2007; 132: 1804-1809.

10. Kapeliovich M, Agmon Y, Zdorovyak A, Hammerman H, Beyar R, Mahamid E, et al. Severe hypoxemia in a patient with acute myocardial infarction. Int J Cardiovasc Intervent 2004; 6: 85-87.
11. Weynand B, Jonckheere A, Frans A, Rahier J. Diabetes mellitus induces a thickening of the pulmonary basal lamina. Respiration 1999; 66: $14-19$.

12. Gu YJ, Mariani MA, van Oeveren W, Grandjean JG, Boonstra PW. Reduction of the inflammatory response in patients undergoing minimally invasive coronary artery bypass grafting. Ann Thorac Surg 1998; 65: 420-424.

13. Asimakopoulos G, Smith PL, Ratnatunga CP, Taylor KM. Lung injury and acute respiratory distress syndrome after cardiopulmonary bypass. Ann Thorac Surg 1999; 68: 1107-1115.

14. Holtz B, Bake B, Sixt R. Prediction of postoperative hypoxemia in smokers and non-smokers. Acta Anaesthesiol Scand 1979; 23: $411-$ 418. 